\title{
The Research on Consumer Decision Process and Problem Recognition
}

\author{
Gao Song ${ }^{1, a}$ \\ ${ }^{1}$ School of Business management, Tonghua Normal University, China \\ adbcy9999@163.com
}

\begin{abstract}
Keywords: consumer decision; nominal decision; limited decision; problem recognition; postpurchase
\end{abstract}

Abstract. This paper examines the nature of the consumer decision process and analyzes the first step in that process - problem recognition. Within problem recognition, we focus on (1) the process of problem recognition, (2) the uncontrollable determinants of problem recognition, and (3) marketing strategies based on the problem recognition process.

\section{Introduction}

The term consumer decision [1-4] produces an image of an individual carefully evaluating the attributes of a set of products, brands, or services and rationally selecting the one that solves a clearly recognized need for the least cost. It has a rational, functional connotation. Consumers do make many decisions in this manner; however, many other decisions involve little conscious effort. Further, many consumer decisions focus not on brand attributes but rather on the feelings or emotions associated with acquiring or using the brand or with the situation in which the product is purchased or used. Although purchases and related consumption behaviour driven by emotional or situational needs have characteristics distinct from the traditional attribute-based model, the decision process model provides useful insights into all types of consumer purchases. We will indicate how it helps us understand emotion-, situation-, and attribute-based decisions. Consumer decisions are frequently the result of a single problem, for example, running low on gasoline. At other times, they result from the convergence of several problems, such as an aging automobile and a growing feeling of inadequacy or low self-esteem. Furthermore, once the decision process begins, it may evolve and become more complex with multiple goals. A consumer noticing a simple need for gas may want to minimize the price paid, avoid one or more brands because of their environmental record, and decide to find a station with food service attached. As the consumer moves from a very low level of involvement with the purchase to a high level of involvement, decision making becomes increasingly complex. While purchase involvements a continuum, it is useful to consider nominal, limited, and extended decision making as general descriptions of the types of processes that occur along various points on the continuum. Keep in mind that the types of decision processes are not distinct but rather blend into each other. Before describing each type of decision process, we must clarify the concept of purchase involvement. We define purchase involvement as the level of concern for, or interest in the purchase process triggered by the need to consider a particular purchase. Thus, purchase involvement is a temporary state of an individual or household. It is influenced by the interaction of individual, product, and situational characteristics. Note that purchase involvement is not the same as product involvement or enduring involvement. A consumer may be very involved with a brand (Starbucks or Saturn) or a product category (coffee or cars) and yet have a very low level of involvement with a particular purchase of that product because of brand loyalty, time pressures, or other reasons.

\section{Nominal Decision Making}

Nominal decision [5-6] making, sometimes referred to as habitual decision making, in effect involves no decision purse. As Figure 1 indicates, a problem is recognized, internal search (long-term memory) provides a single preferred solution (brand), that brand is pur- chased, and an evaluation occurs only if the brand fails to perform as expected. Nominal decisions occur when there is very low involvement with the purchase. 


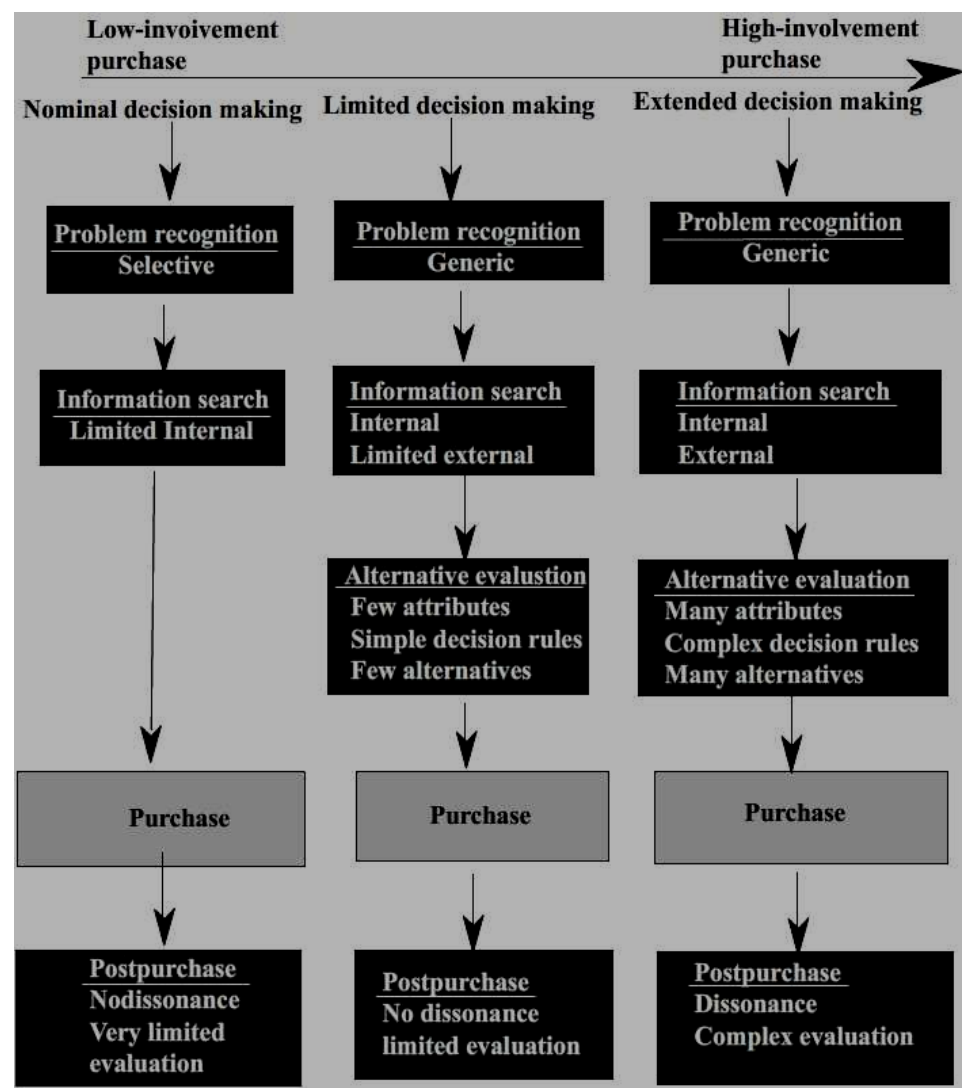

Fig. 1 -Involvement and types of decision making

A completely nominal decision does not even include consideration of the "do not purchase" alternative. For example, you might notice that you are nearly out of Aim toothpaste and resolve to purchase some the next time you are at the store. You don't even consider not replacing the toothpaste or purchasing another brand. At the store, you scan the shelf for Aim and pick it up without considering alternative brands, its price, or other potentially relevant factors. You are brand loyal. It will be very difficult for a competitor to gain your patronage. In this example, you have a fairly high degree of product involvement but a low degree of purchase involvement because of your brand loyalty. Should you encounter a challenge to the superiority of Aim, perhaps through a news article, you would most likely engage in a high-involvement decision process before changing brands. Repeat Purchases In contrast, you may believe that all ketchup is about the same and you may not attach much importance to the product category or purchase. Having tried Del Monte and found it satisfactory, you now purchase it whenever you need ketchup. Thus, you are a repeat purchaser of Del Monte ketchup, but you are not committed to it. Should you encounter a challenge to the wisdom of buying Del Monte the next time you need ketchup, perhaps because of a point-of-sale price discount, you would probably engage in only a limited decision process before deciding on which brand to purchase.

\section{Limited Decision Making}

Limited decision [7-8] making involves internal and limited external search, few alternatives, simple decision rules on a few attributes, and little post purchase evaluation. It covers the middle ground between nominal decision making and extended decision making. Limited decision making also occurs in response to some emotional or situational needs. As Figure 1 illustrates, problem recognition is the first stage of the decision process. We will describe this stage and discuss the marketing applications associated with it in the balance of this paper. We devote the remaining four stages of the consumer decision process and discuss the relevant marketing applications. Our discussion of the decision process is based primarily on studies conducted in America. Although the evidence is limited, it appears that consumers in other cultures use similar processes. 


\section{The nurture of problem of recognition}

Problem recognition [9-10] is the first stage in the consumer decision process. Problem recognition is the result of a discrepancy between a desired state and an actual state that is sufficient to arouse and activate the decision process. An actual state is the way an individual perceives his or her feelings and situation to be at the present time. A desired state is the way an individual wants to feel or be at the present time. For example, you probably don't want to be bored on Friday night. If you find yourself alone and becoming bored, you would treat this as a problem because your actual state (being bored) and your desired state (being pleasantly occupied) are different. You could then choose to watch a television program, rent a video, call a friend, go out, or take a wide array of other actions.

Without recognition of a problem, there is no need for a decision. This condition is shown in Figure 2, when there is no discrepancy between the consumer's desired state (what the consumer would like) and the actual state (what the consumer perceives as already existing). Thus, if Friday night arrives and you find yourself engrossed in a novel, your desire to be pleasantly occupied (desired state) and your condition of enjoying a novel would be consistent, and you would have no reason to search for other activities.

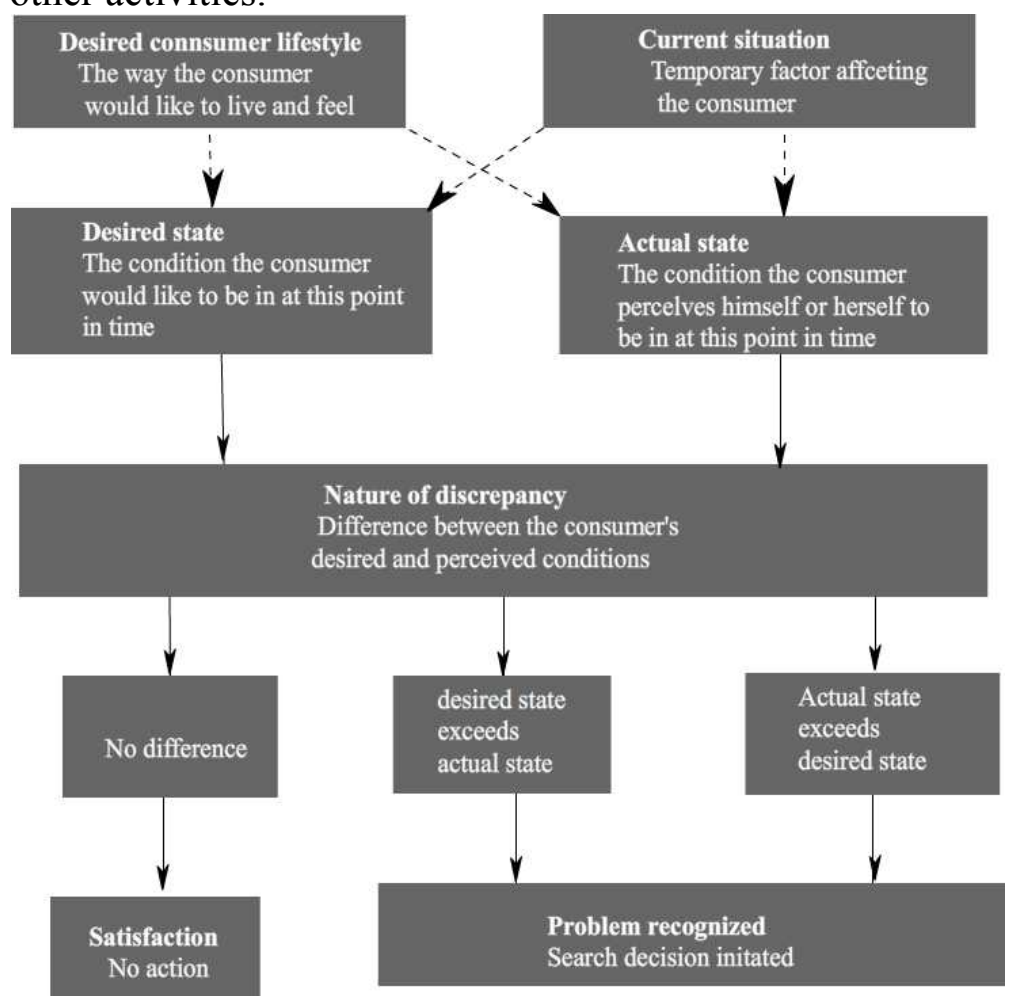

Fig. 2 - The process of problem recognition

On the other hand, when there is a discrepancy between a consumer desire and the perceived actual state, recognition of a problem occurs. Figure 2 indicates that any time the desired state is perceived as being greater than or less than the actual state, a problem exists. For example, being pleasantly occupied (desired state) would generally exceed being bored (actual state) and result in problem recognition. However, if your roommate suddenly showed up with a rowdy party, you might find yourself with more stimulation (actual state) than the medium level you actually desire. This too would result in problem recognition. In Figure 2, consumer desires are shown to be the result of the desired lifestyle of the consumer and the current situation. Thus, a consumer whose self-concept and desired lifestyle focus on outdoor activities will desire frequent participation in such activities. A current situation of new snow in the mountains or warm weather at the beach would tend to increase that person's desire to be engaged in outdoor sports. Perceptions of the actual state are also determined by a consumer's lifestyle and current situation. Consumers' lifestyles are a major determinant of their actual state because that is how they choose to live given the constraints imposed by their resources. Thus, a consumer who has chosen to raise a family, have signifi cant material possessions, and pursue a demanding career is likely to have little free time for outdoor activities 
(actual state). The current situation - a day off work, a big project due, or a sick child — also has a major impact on how consumers perceive the actual situation.

\section{Summary}

Consumer decision making becomes more extensive and complex as purchase involvement increases. The lowest level of purchase involvement is represented by nominal decisions: A problem is recognized, long-term memory provides a single preferred brand, that brand is purchased, and only limited post purchase evaluation occurs. As one move from limited decision making toward extended decision making, information search increases, alternative evaluation becomes more extensive and complex, and post purchase evaluation becomes more thorough.

\section{Acknowledgements}

The work was supported by Research Foundation of Education Bureau of Jilin Province (Grant No.2014388)

\section{References}

[1]Vitalija Butkevičienè, Jurgita Stravinskienè, Aušra Rūtelionè, Impact of Consumer Package Communication on Consumer Decision Making Process, Engineering Economics, 1 , 2008, 57.

[2]Carolyn Yoon, Richard Gonzalez, Antoine Bechara, Decision neuroscience and consumer decision making, Marketing Letters, 23 (2), 2012, 473-485.

[3]Shih-Chieh Chuang, Hung-Ming Lin, The Effect of Induced Positive and Negative Emotion and Openness-to-Feeling in Student's Consumer Decision Making, Journal of Business and Psychology, 22 (1), 2007, 65-78.

[4]Sadaaki Fukui, Michelle P. Salyers, Marianne S. Matthias, Predictors of Shared Decision Making and Level of Agreement Between Consumers and Providers in Psychiatric Care, Community Mental Health Journal, 50 (4), 2014, 375-382.

[5]Robert L. Armacost, Jamshid C. Hosseini, Julie Pet-Edwards, Using the Analytic Hierarchy Process as a Two-phase Integrated Decision Approach for Large Nominal Groups, Group Decision and Negotiation, 8 (6), 1999, 535-555.

[6]Eviatar Z, Zaidel E, Wickens T, Nominal and physical decision criteria in same-different judgments, Perception \& psychophysics, 56 (1), 1994, 62-72.

[7]Sanghwa Jeong, Yonghun Oh, Sangsuk Kim, Robust Control of multi-echelon production-distribution systems with limited decision policy (II), KSME International Journal, 14 (4), 2000, 380-392.

[8]Reuland Daniel S, Ko Linda K, Fernandez Alicia, Braswell Laura C, Pignone Michael, Testing a Spanish-language colorectal cancer screening decision aid in Latinos with limited English proficiency: Results from a pre-post trial and four month follow-up survey, BMC Medical Informatics and Decision Making, 12 (1), 2012, 53.

[9]Glass J E, Grant J D, Yoon H Y, Bucholz K K, Alcohol problem recognition and help seeking in adolescents and young adults at varying genetic and environmental risk, Drug and alcohol dependence, 153 , 2015, 250-7.

[10]A. V. Kel'manov, S. A. Khamidullin, A recognition problem for a vector alphabet generating a sequence with a quasiperiodic structure, Numerical Analysis and Applications, 2 (3), 2009, 220-229. 